\title{
Oxytocin weakens self-other distinction in males during empathic responses to sadness: An event-related potentials study
}

\author{
Tong Yue ${ }^{\text {Corresp., } 1}$, Ying Xu ${ }^{1}$, Liming Xue $^{1}$, Xiting Huang ${ }^{1}$ \\ ${ }^{1}$ Faculty of Psychology, Southwest University, Chongqing, China \\ Corresponding Author: Tong Yue \\ Email address: yuetong87@swu.edu.cn
}

By making use of event-related potential (ERP) technology, a randomized, double-blind, between-subject design study was performed in order to investigate whether OXT can weaken men's self-other distinction during empathic responses to sad expressions. In the two experimental tasks, 39 male subjects were asked to either evaluate the emotional state shown in a facial stimulus (other-task) or to evaluate their own emotional responses (self-task). The results revealed that OXT reduced the differences in P2 (150-200 ms) amplitudes between sad and neutral expressions in the self-task but enhanced P2 to sad expressions in the other-task, indicating OXT's role in integrating the self with others instead of separating them. In addition, OXT also reduced the LPC (400-600 ms) amplitudes between sad-neutral expressions in the self-task, implying that OXT's weakening effects on the self-other distinction could occur at both the early and late cognitive control stages of the empathic response. 
1

2 Oxytocin Weakens Self-Other Distinction in Males 3 during Empathic Responses to Sadness: An Event4 Related Potentials Study

5

6

7

8

9

10

11

22

23

24

25

26

27

28

29

30

31

32

33

34

35

36

37

38

39
Tong Yue ${ }^{1}$, Ying $\mathrm{Xu}^{1}$, Liming $\mathrm{Xue}^{1}$, Xiting Huang ${ }^{1}$

${ }^{1}$ Faculty of Psychology, Southwest University, Chongqing, China

Corresponding Author:

Tong Yue

No. 2, Tiansheng Road, Beibei, Chongqing 400715, China

Email address: yuetong87@swu.edu.cn 
41

42

43

44

45

46

47

48

49

50

51

52

53

54

55

56

57

58

59

60

61

62

63

64

65

66

67

68

69

70

71

72

73

74

75

76

77

78

79

\section{Abstract}

By making use of event-related potential (ERP) technology, a randomized, double-blind, betweensubject design study was performed in order to investigate whether OXT can weaken men's selfother distinction during empathic responses to sad expressions. In the two experimental tasks, 39 male subjects were asked to either evaluate the emotional state shown in a facial stimulus (othertask) or to evaluate their own emotional responses (self-task). The results revealed that OXT reduced the differences in P2 (150-200 ms) amplitudes between sad and neutral expressions in the self-task but enhanced P2 to sad expressions in the other-task, indicating OXT's role in integrating the self with others instead of separating them. In addition, OXT also reduced the LPC (400-600 ms) amplitudes between sad-neutral expressions in the self-task, implying that OXT's weakening effects on the self-other distinction could occur at both the early and late cognitive control stages of the empathic response.

\section{Introduction}

Humans are social animals that rely strongly on social relations and interactions. The neuropeptide oxytocin (OXT), which is an ancient and conserved hormone, has been shown to influence many different aspects of social cognition and behavior in humans (Baumgartner, Heinrichs, Vonlanthen, Fischbacher, \& Fehret, 2008; Cardoso, Linnen, Joober, \& Ellenbogen, 2012; Kumsta \& Heinrichs, 2012; Quirin Kuhl, \& Düsing, 2011; Sauer, Montag, Reuter, \& Kirsch, 2013). One particular concern, found in an increasing number of studies, is OXT's potential role in influencing self-processing, especially the distinction between self and other. Some research have shown that OXT may sharpen the self-other percept by increasing the ability to discriminate between own and unfamiliar faces, as observed during a morphing paradigm (Colonnello, Chen, Panksepp, \& Heinrichs, 2013). On the other hand, other studies have indicated that OXT could blur the self-other distinction. For example, Zhao et al. (2016) found that OXT reduces self-bias in self and other trait judgments. This was reflected as reduced response times and accuracy of subsequent recall at the behavioral level, as well as reduced responses of the medial prefrontal cortex (mPFC) and its functional connectivity with the default mode network (DMN), which is involved in self-processing. All in all, the question about whether OXT blurs the distinction between self and other is still under debate.

Empathy is an emotional state that results from the observation, imagination, and inference of emotions of other people, in conjunction with an awareness that a person's emotions originate within themselves (De Vignemont \& Singer, 2006). The distinction between the self and others is regarded as an important cognitive component and emotional regulation strategy of empathy progression (Decety \& Jackson, 2004). Whether from anecdotal observation of people's daily lives or from experimental research, men express significantly lower empathy than women (O'Brien, Konrath, Gruhn, \& Hagen, 2013; Suh, Hong, Lee, Gonnella, \& Hojat, 2012), and some researchers believe that a higher tendency toward the self-other distinction may account for the lower empathy 
80

81

82

83

84

85

86

87

88

89

90

91

92

93

94

95

96

97

98

99

100

101

102

103

104

105

106

107

108

109

110

111

112

113

114

115

116

117

118

119

among men. For example, in the study by Schulte-Rüther et al. (2008), participants were asked to perform either the self-task (evaluate one's affective responses to facial stimuli) or the other-task (focus on and evaluate the emotional states of facial expressions). The results showed that male subjects recruited the left temporoparietal junction (TPJ) more strongly compared with females in the self-task and that the TPJ is one component of a neural circuitry that plays a distinct role in self-other distinction (Decety \& Sommerville, 2003; Jackson, Brunet, Meltzoff, \& Decety, 2006). Using similar self-tasks and other-tasks, Luo et al.'s (2015) event-related potentials (ERPs) study also reported that only males (but not females) exhibited an elevated P2 deflection when faced with sad expressions than neutral facial stimuli during the self-task; however, the discrepancy was absent during the other-task. According to the results, Luo et al. (2015) deduced that males might be inclined to distinguish the self from others during the progress of empathy, blocking their resonation with another individual's emotions. Therefore, the characteristics of male psychological processing with stronger self-other distinction in empathy progress provide a natural opportunity to test OXT's role in influencing self-processing: if OXT can effectively decrease the tendency toward self-other distinction in men, there would be an enhancing effect on empathy. On the other hand, OXT could weaken empathy in men or remain the same.

In fact, Abu-Akel, Palgi, Klein, Decety, and Shamay-Tsoory (2015) have previously discussed the effects of OXT on empathy using the affective perspective-taking task, showing that OXT could increase empathy for pain in male subjects in the other-task instead of the self-task. According to the results, Abu-Akel et al. (2015) speculated that OXT may separate, rather than integrate, self and others in empathic responses. However, it may not be a compelling conclusion for several reasons. First, the indicator was not precise. Abu-Akel et al. (2015) adopted subjective reporting to measure the degree of empathic responses, but it was difficult to fully capture the differences in cognitive processing caused by variable manipulation solely under participants' introspection. Not only that, Pfundmaira, Rimpela, Duffyb, and Zwarg (2018) found that OXT could blur the distinction between self and other only implicitly (e.g. reflexes and brain imaging) and not explicitly (e.g. ratings and self-report measures). Second, OXT's influence on increasing empathic responses when taking an other-perspective, but not a self-perspective, does not necessarily demonstrate its role in enhancing the ability to recognize differences between self and others. Using the visual perspective-taking task, Yue, Jiang, Yue, and Huang (2017) reported that OXT can reduce self-centeredness tendencies and increase the perceptiveness of others. Thus, Abu-Akel et al.'s (2015) results can also be attributed to OXT's effects of promoting the transition from the self-to-other perspective and thus, integrate the self with others instead of separating them. Therefore, it is necessary to verify this issue with more reliable and effective indicators.

Based on the above discussion, the present study assumes that OXT can effectively decrease the tendency toward self-other distinction in male empathic responses. In order to further determine whether the weakening effect occurs in the early stage of the empathic response's automatic processing or in the later stage of cognitive regulation, the present study adopts ERPs with higher sensitivity and time resolution. According to previous studies, the early P2 component and the late LPC component, which are associated with both self-related information (Liu, Sheng, 
120 Woodcock, \& Han, 2013) and processing of affective information (Sheng \& Han 2012; Sheng, 121 Liu, Li, Fang, \& Han, 2014), can be effective indicators of the variation of self-other distinction in 122 male participants' empathic responses. For example, it was found that P2 may reflect the gender 123 difference in self-other distinction ability by the fact that sad faces induced larger P2 amplitudes in the self-task in men but not in women, since they have a greater tendency than women to automatically focus on and monitor their own effect in response to distress cues in the early stage of empathic responses (Luo et al., 2015). Therefore, the first hypothesis of this study is, if OXT can decrease the male tendency toward self-other distinction in the early stage of the empathic response, the P2 discrepancy amplitude induced by the sad faces in the self-task should be absent, similar to that of female participants in the study of Luo et al. (2015). In addition, LPC is an important indicator of self-other distinction ability for men, as they produced greater differential LPC amplitudes towards sad faces during the self-task compared with other-task, while women did not (Luo et al., 2015). This is presumably because sadness can also communicate a request for help and elicit an approach and other-related prosocial motivations (Seidel, Habel, Kirschner, Gur, \& Derntl, 2010), whereas men are more likely to allocate attentional resources toward selfrelated affective information caused by sad faces than women, which may underlie the gender difference of the LPC amplitude. Thus, if the effects of OXT last until the late processing stage of empathic responses, we can see signs from the LPC component, such as decreasing LPC amplitudes induced by sad faces in the self-task, which constitutes the second hypothesis of this study.

With reference to the previous article (Luo et al., 2015; Schulte-Rüther et al., 2008), we employed two emotional perspective tasks using sad facial expressions to induce empathic responses in both the self-task and other-task. The present study employed a double-blind randomized controlled design, in which male participants in both the OXT and placebo (PLC) groups completed the emotional perspective tasks. By examining the influence of OXT on the P2 and LPC components of the empathic responses, this study explored whether it could decrease the male tendency toward self-other distinction in empathic responses.

\section{Materials \& Methods}

\section{Participants and Treatment}

Forty male undergraduate students (mean age $=20.65 \pm$ S.D. 1.85) were recruited via advertisements from Southwest University in China. Of these 40, one subject withdrew at the drug application stage because of rhinitis, leaving 39 subjects in total. Before the experiment, each subject provided written informed consent. All the subjects were asked to maintain their regular sleep patterns leading up to the formal experiment and to abstain from alcohol, caffeine, and smoking for at least 12 hours. No participant had reported any neurological or psychiatric problems and none were taking any form of medication. The Ethics Committee of Southwest University approved the current study (IRB NO.H18007), and the procedures involved are in line with the Declaration of Helsinki. 
160

161

162

163

164

165

166

167

168

169

170

171

172

173

174

175

176

177

178

179

180

181

182

183

184

185

186

187

188

189

190

191

192

193

194

195

196

197

198

199

We used a double-blind, controlled, and between-subject design in this study. First, each subject was given a single intranasal administration of 24 IU OXT or PLC. The OXT was administered in the form of a Syntocinon spray, produced by Sichuan Meike Pharmacy Co. Ltd, China; 3 puffs of 4 IU per nostril, with 30 seconds between each puff. The PLC, also produced by Meike Pharmacy Co. Ltd., comprised the same ingredients with the exception of the neuropeptide and was packaged in an identical bottle. Striepens et al. (2013) suggested that the formal experiment should be started 45 minutes after administration of the OXT or PLC treatments because the peptide concentration would increase in the cerebrospinal fluid during this period. Ultimately, 20 men were treated with OXT, and the remaining 19 were treated with PLC (see Figure 1). In the post-experiment interview, the subjects were unable to identify which treatment they had received (with any degree of accuracy better than by chance).

In order to control for the potential confounding effect of trait empathy between the OXT and PLC groups, before the start of the experiment, all subjects needed to complete an inter-personal reactivity index (IRI)-C (Zhang et al., 2010), which includes four sub-scales: fantasy (FS), perspective taking (PT), personal distress (PD), and empathic concern (EC).

\section{INSERT FIGURE 1 HERE}

\section{Experimental Design}

From the Chinese Affective Picture System (CAPS), we selected 30 sad and 30 neutral facial expressions, of which each category was divided evenly between males and females (Bai, Ma, Huang, \& Luo, 2005). According to the CAPS scores, t-test comparisons revealed significant differences between sad and neutral emotional faces on both valence $(M \pm S D$ : sad $=2.87 \pm 0.68$, neutral $=4.63 \pm 0.79, t(58)=-14.26, p<0.001)$ and arousal $(\mathrm{sad}=5.68 \pm 1.13$, neutral $=3.70 \pm$ $1.10, t(58)=7.62, p<0.001)$. All expressions were identical in size and resolution $(15 \mathrm{~cm} \times 10$ $\mathrm{cm}, 100$ pixels per inch). The pictures were presented at the center of a 17-inch CRT display, approximately 1 meter in front of the subjects.

In the current study, we performed two emotional perspective tasks, which included both a self-task and an other-task. In the other-task, we told the subjects to press the F button if the emotion expressed by the face was sad and the $\mathrm{J}$ button if neutral. The order of the $\mathrm{F}$ (sad) and $\mathrm{J}$ (neutral) keys was counterbalanced between participants. The procedure of the self-task was similar to that of the other-task, except that we asked the subjects to evaluate their own emotional reaction when observing the presented faces in the experiment. The subjects were emphatically told that there were absolutely no right or wrong answers and instructed to make choices according to their own feelings.

Every task had two blocks, each containing 60 trials (30 emotional and 30 neutral faces). The order of the two tasks was randomized across subjects. After completing each block, the participants were permitted to rest. Before the formal experiment, the subjects were instructed to familiarize themselves with the experimental procedure via practice trials. The trial in the formal 
200

201

202

203

204

205

206

207

208

209

210

211

212

213

214

215

216

217

218

219

220

221

222

223

224

225

226

227

228

229

230

231

232

233

234

235

236

237

238

239

experiment was conducted as follows: First, a red fixation "+" lasting $500 \mathrm{~ms}$ was presented on the black screen. Then, a blank screen was shown for 500 to $800 \mathrm{~ms}$, followed by pictures of either sad or neutral faces, which were presented in random order. The subjects were asked to respond according to the requirements of the task as quickly and accurately as possible, and once the subject pressed a key, the facial expression on the screen was terminated. If no response was made, the visual stimulus disappeared automatically after $2000 \mathrm{~ms}$.

\section{EEG recording and data processing}

The EEG data were recorded through 64 scalp sites with tin electrodes mounted in an elastic cap (Brain Products, Munich, Germany). The horizontal and vertical electrooculograms (EOGs) were recorded from electrodes placed above and at the outer canthus of the right eye to control for eye movement artifacts. The ground electrode was on the medial frontal aspect and the references were on the left and right mastoids. The EEG and EOGs were amplified with a 0.05 to $100 \mathrm{~Hz}$ bandpass filter and a $500 \mathrm{~Hz}$ sampling rate. The electrode impedances were kept below $10 \mathrm{k} \Omega$. Subjects were asked to stay still, avoid blinking, relax facial muscles, and look at the fixation cross on the screen.

We used the Vision Analyzer 2.1 (Brain Products, Gilching, Germany) to perform the offline analyses. The data were segmented into $1200 \mathrm{~ms}$ durations, including $200 \mathrm{~ms}$ of prestimulation baseline recording. The regression analysis was performed to remove the EOGs with a band pass filter of 0.01-30 Hz. The trials excluded from the averaging included trials with artifact contamination due to amplifier clipping and EOG artifacts (average EOG voltage exceeding \pm 80 $\mathrm{mV}$ ), bursts of electromyography activity, and peak-to-peak deflection exceeding $\pm 80 \mathrm{mV}$.

Based on visual observation, as well as on previous studies (Sheng et al., 2012), we analyzed the mean amplitude of the 150 to $200 \mathrm{~ms}$ period after the onset of the outcome for the P2, and 400 to $600 \mathrm{~ms}$ for the LPC. The P2 was mainly distributed in the frontal lobe and central regions, while LPC was widely distributed on the scalp. Therefore, we included the mean amplitudes of electrodes located in the frontal $(\mathrm{F} 3, \mathrm{~F} 4, \mathrm{Fz}, \mathrm{FC} 3, \mathrm{FC} 4, \mathrm{FCz})$ and central $(\mathrm{C} 3, \mathrm{C} 4, \mathrm{Cz})$ regions for the P2 analysis, and the frontal (F3, F4, Fz, FC3, FC4, FCz), central (C3, C4, Cz), central parietal (CP3, CP4, CPz), and parietal (P3, P4, Pz) regions for the LPC analysis. Regarding the effect of laterality, we also took into account the electrodes on the left hemisphere (F3, FC3, C3, CP3, P3), central line (Fz, FCz, Cz, CPz, Pz), and the right hemisphere (F4, FC4, C4, CP4, P4) for the LPC analysis.

A mixed-design ANOVA was performed on the mean amplitudes of the P2 and LPC with brain regions, laterality (left hemisphere, central line and right hemisphere), facial expression (sad and neutral) and task (self and other) as the within-subjects factors and treatment type (OXT vs. PLC) as the between-subjects factor. The data were analyzed via SPSS 16.0 using the GreenhouseGeisser correction when the sphericity hypothesis was violated, and Bonferroni correction was applied for multiple comparisons. 
240

241

242

243

244

245

246

247

248

249

250

251

252

253

254

255

256

257

258

259

260

261

262

263

264

265

266

267

268

269

270

271

\section{Results}

\section{Behavioral results}

The independent $t$-test of IRI-C scores showed that there was no significant difference between the OXT and PLC groups, regardless of the four sub-dimensions or the total dimensions (all $p>0.51)$.

Accuracy analysis revealed a significant main effect of task type $\left(F_{1,37}=47.72, p<0.001\right.$, $\left.\eta_{p}^{2}=0.56\right)$, with significantly greater accuracy during the other-task $(\mathrm{M} \pm \mathrm{SD}: 89.43 \pm 9.47 \%)$ than during the self-task $(68.44 \pm 20.93 \%)$. Another significant main effect of facial expression $\left(F_{1,37}=11.20, p<0.01, \eta_{p}^{2}=0.23\right)$ was discovered, showing that accuracy for the neutral faces $(84.20 \pm 22.00 \%)$ was significantly higher than for the sad faces $(73.67 \pm 18.19 \%)$. No other main effects or interactions were found to significantly impact the degree of accuracy. The ANOVA of response time revealed a significant main effect of task type $\left(F_{1,37}=15.90, p<0.001, \eta^{2}{ }_{p}=0.30\right)$, with RTs being slower in the self-task $(1190.48 \pm 246.97 \mathrm{~ms})$, as compared to the other-task $(990.66 \pm 190.84 \mathrm{~ms})$. The main effect of emotional types on response time was also significant $\left(F_{1,37}=9.92, p<0.01, \eta_{p}^{2}=0.21\right)$, with longer RTs for sad expressions, as compared with neutral expressions $(1142.09 \pm 237.19$ vs $1039.05 \pm 232.47 \mathrm{~ms})$. No other main effects or interaction were found in the response time analysis.

\section{ERP Results}

\section{INSERT TABLE 1 HERE}

\section{P2}

The ANOVA of the mean amplitudes of the P2 component suggested a main effect of affect $\left(F_{1,37}=4.11, p=0.05, \eta_{p}^{2}=0.10\right)$, with significantly larger amplitudes induced by sad faces $(3.44$ $\pm 0.49 \mu \mathrm{V})$ than by neutral faces $(2.93 \pm 0.52 \mu \mathrm{V})$. There was also a significant interaction effect involving treatment type, task, and facial expression $\left(F_{1,37}=5.99, p<0.05, \eta^{2}{ }_{p}=0.14\right)$. Results of the simple effects test showed that sad expressions $(3.05 \pm 0.79 \mu \mathrm{V})$ elicited larger $\mathrm{P} 2$ amplitudes than did neutral expressions $(1.79 \pm 0.80 \mu \mathrm{V})$ in the placebo group self-task $\left(F_{1,37}=5.84, p<0.05\right.$, 
$\left.272 \eta_{p}^{2}=0.14\right)$, but that there was no significant difference between the two facial expressions in the 273 other-task. For the OXT group, sad faces $(4.21 \pm 0.70 \mu \mathrm{V})$ induced larger P2 amplitudes than did 274 neutral faces $(3.11 \pm 0.77 \mu \mathrm{V})$ in the other-task $\left(F_{1,37}=4.32, p<0.05, \eta_{p}^{2}=0.10\right)$, while there was 275 no difference in the self-task. In addition, significant interaction effects were found between brain 276 area, laterality, treatment type, task, as well as facial expression (see Table 1). Further analysis 277 showed that under the influence of OXT, sad faces elicited larger P2 amplitudes in the left central 278 (C3) region in the other-task, while neutral faces induced larger P2 amplitudes in the left and right 279 left central (C3, C4) regions in the self-task (see Figure 2).

280

281

INSERT FIGURE 2 HERE

282

283

284

285

286

287 showed no obvious difference between the two groups in the other-task, except that the P2 of the 288 OXT group was significantly smaller than that of the PLC group in the self-task $\left(F_{1,37}=5.51, p<\right.$ $0.05, \eta_{p}^{2}=0.13$ ) (see Figure 3).

289

290

291

INSERT FIGURE 3 HERE

292

293

294

295

LPC

296

ANOVAs of the difference waves at the P2 component revealed a significant interaction effect between treatment type and task $\left(F_{1,37}=5.52, p<0.05, \eta_{p}^{2}=0.13\right)$, and the simple effects test OXT group was significantly smaller than that of the PLC group in the self-task $\left(F_{1,37}=5.51, p<\right.$

297

The analyses of ERP amplitudes focused on the LPC component showed that the main effects of facial expression, brain region, and laterality were all significant $\left(F_{1,37}=48.24, p<0.001, \eta^{2} p\right.$ 298 $=0.56 ; F_{1,37}=63.18, p<0.001, \eta_{p}^{2}=0.62 ; F_{1,37}=7.59, p=0.001, \eta^{2}{ }_{p}=0.17$ ) (see Table 1). Compared 299 with neutral expressions, sad expressions induced larger LPCs $(8.82 \pm 0.73 \mu \mathrm{V}$ vs $6.59 \pm 0.73 \mu \mathrm{V})$. The amplitudes from the frontal-central to the parietal regions gradually decreased, and the 301 amplitudes over the midline were larger than those of the left and right sides. Additionally, there 302 was a significant three-way interaction for facial expression, task, and treatment type: for 303 participants in the OXT group, larger LPC amplitudes were elicited by sad expressions, as 
304 compared with neutral faces in the other-task $(8.30 \pm 1.02 \mu \mathrm{V}$ vs $6.01 \pm 1.05 \mu \mathrm{V}, p<0.01)$, but 305 with no significant difference in the self-task; for participants of the PLC group, sad expressions 306 induced larger LPC amplitudes than neutral expressions in both the other-task $(8.96 \pm 1.07 \mu \mathrm{V}$ vs

307

308

309

310

311

312

313

314

315

316

317

318

319

320

321

322

323

324

325

326

327

328

329

330

331

332

333

334

$6.97 \pm 1.10 \mu \mathrm{V})$ and the self-task $(8.82 \pm 1.45 \mu \mathrm{V}$ vs $5.08 \pm 1.16 \mu \mathrm{V} ; p<0.01)$. Additionally, a significant four-way interaction for treatment type, task, brain region, and facial expression was also found $\left(F_{1,37}=2.83, p<0.05, \eta^{2}{ }_{p}=0.07\right)$, and further analysis indicated that under the impact of OXT, the LPC amplitudes induced by neutral expressions in the frontal regions were the largest.

The analysis of the difference waves at the LPC component revealed that the interaction effect between group and task was significant $\left(F_{1,37}=5.99, p<0.05, \eta_{p}^{2}=0.14\right)$. Further, the simple effects test showed that there was no significant difference between the two treatment types in the other-task, but smaller difference waves were found in the OXT group than in the PLC group in the self-task $\left(F_{1,37}=13.73, p=0.001, \eta_{p}^{2}=0.28\right)$ (see Figure 4).

(1)

(1)

\section{INSERT FIGURE 4 HERE}

(1)

\section{Discussion}

In the present study, we explored whether OXT can weaken men's self-other distinction in the empathic responses during emotional perspective tasks. Although there was limited evidence at the behavioral level, at the more sensitive neural level, we found differences between the OXT and PLC groups reflected in the P2 and LPC components, which provided evidence that OXT blurs self-other distinction.

At the behavioral level, the difference in accuracy or reaction time between the OXT and PLC group during the emotional perspective tasks was not significant, which is consistent with previous studies (Luo et al., 2015; Jin et al. 2013; Schulte-Rüther et al. 2008). Previous studies have also suggested that OXT could blur the self-other distinction only in an implicit response but not at an explicit level (Pfundmaira et al., 2018). This may be because the explicit response is conscious, requires cognitive resources, and may even be superimposed by conscious thoughts or selfpromotion, while the implicit response is not (Bargh, 1994). Thus, the explicit behavioral 
335 indicators used may be less sensitive to the effects of OXT, and the findings of this study are 336 mainly reflected at the level of implicit neural responses.

337 The P2 results of the PLC group validated the conclusion of Luo et al. (2015), who conducted 338 tests on males and also reported that sad facial stimuli elicited larger amplitudes than did neutral 339 facial stimuli during the self-task, but the discrepancy was absent during the other-task. According 340 to previous studies, the P2 component is sensitive to salient stimuli (Carretié et al. 2001; Huang \& 341 Luo, 2006; Yuan et al. 2007) and is therefore considered to be associated with early automatic 342 affect sharing during empathic responses (Sheng \& Han 2012; Sheng et al. 2014); on the other 343 hand, it might also reflect the attentional effect in self-relevant information (Liu et al. 2013). Thus, 344 the larger P2 amplitudes elicited by sad than neutral facial stimuli during the self-task in the PLC 345 group may reflect the attentional enhancement caused by self-relevance processing in the early 346 stage, which suggests that males are likely to spontaneously adopt the self-perspective, and attend 347 more to their own feelings, despite the origin of these feelings from others. Therefore, we suggest 348 that men's reactions to their own emotions in P2 may reflect their differences between themselves 349 and others, which echoes the findings by Schulte-Rüther et al. (2008).

350 These results support our first hypothesis that male participants in this study would show a 351 trend consistent with female participants in the study of Luo et al. (2015) after OXT usage; that is 352 to say, although sad facial stimuli induced larger P2 compared to neutral facial stimuli during the 353 other-task, the difference between these two expressions disappeared in the self-task. Further 354 analysis on the differential amplitudes showed that, compared with the PLC group, OXT 355 significantly reduced the $\mathrm{P} 2$ differentiation between the expressions in the self-task but tended to 356 357 increase differences in P2 in the other-task. Although the weakening of the differential wave in the self-task could be attributed to decreasing brain reactivity to negative emotions and increasing neural activity involved in emotion regulation (Ma et al., 2016), it is difficult to explain the opposite trends in the other-task. Therefore, the more likely reason is OXT's established role in 360 decreasing self-interest and increasing interest in others (Yue et al., 2017) that is reflected in the present study by a shift in male participants' focus from self to others, which weakens their 362 emotion monition and regulation in response to facial expressions, leading to a differentiation between the facial stimuli at P2 arising in the other-task. In other words, OXT may play a role in attenuating the distinction between the self and other during the early automatic stage of the 365 empathic response. 
366

367

368

369

370

371

372

373

374

375

376

377

378

379

380

381

382

383

384

385

386

387

388

389

390

391

392

393

394

395

396

As for the LPC component, the results of the PLC group revealed that the mean amplitudes induced by the sad expressions were greater than those elicited by the neutral facial stimuli in both the self- and the other-task; however, for the OXT group, the difference between the sad and neutral expressions was only significant in the other- but not in the self-task. Further analysis on the difference waves of the LPC showed that the self-task was significantly smaller in the OXT group than in the PLC group, but not in the other-task. Similar to the P2 component, the LPC component is believed to reflect the attention distribution processing of emotion-related stimuli (Li, Li, Chen, \& Li, 2012; Schupp et al. 2000; Van Strien, De Sonneville, \& Franken, 2010) and may be related to the intrinsic motivational significance of self-related information (Fields \& Kuperberg 2012; Li \& Han 2010). Given that after inhaling OXT, the discrepancy between sad and neutral expressions remained only during the other-task but not the self-task, the OXT's effect cannot be attributed to downregulation of emotionally relevant stimuli (Ma et al., 2016) but rather to its effects of reduced self-orientation. Thus, the above results validate the present study's hypothesis that the weakening effect of OXT on men's self-other distinction still exists in the later stage of the empathic response.

Several limitations may still exist in our current study, which can be addressed in future studies. First, the current study only used sad and neutral faces as the empathy inducing stimulus. Thus, the subjects simply needed to choose one emotion. This design cannot explain whether the OXT effects observed in this study only apply to sad-specific emotions, general negative emotions, or whether they also apply to positive emotions. Future research should investigate whether other types of emotions could replicate the same OXT effects. Moreover, differences in sex are an important factor when examining the effects of OXT on human social cognition (Fischer-Shofty, Levkovitz, \& Shamay-Tsoory, 2013; Preckel, Scheele, Kendrick, Maier, \& Hurlemann, 2014; Theodoridou, Penton-Voak, \& Rowe, 2013). Thus, the potentially sexually dimorphic effects of OXT on the self-other distinction during empathy should be considered in future studies. Our conclusion that OXT weakens the self-other distinction during empathic responses was, to a large extent, based on indirect inferences. Future researchers could select more direct indicators (e.g. the activity of self-related brain areas), to further reveal the influence of OXT. Ultimately, the sample size in the current study is relatively small and we did not measure affective state/mood or the awareness of one's own emotions (self-awareness/alexithymia), which may affect the results of this study to some extent. Addressing these deficiencies would be an important avenue for future 
397

398

399

400

401

402

403

404

405

406

407

408

409

410

411

412

413

414

415

416

417

418

419

420

421

422

423

424

425

426

427

428

429

430

431

research.

\section{Conclusions}

The results showed that OXT reduces the differences between neural responses to sad and neutral expressions in the self-task but enhances neural responses to sad expressions in the othertask during the early stage of the empathic response, which provides evidence that OXT appears to blur the distinction between self and other in the context of decreasing self-interest and increasing interest in others. In addition, OXT's effects of reducing the neural differences between sad and neutral expressions in the self-task persisted in the later cognitive control stage, which implies that the role of OXT in weakening the self-other distinction seems to occur throughout all stages of the empathic response.

\section{References}

Abuakel, A., Palgi, S., Klein, E., Decety, J., \& Shamaytsoory, S. (2015). Oxytocin increases empathy to pain when adopting the other- but not the self-perspective. Social Neuroscience, 10(1), 7-15.

Bai, L., Ma, H., Huang, Y. X., \& Luo, Y. J. (2005). The development of native Chinese affective picture system-a pretest in 46 college students. Chinese Mental Health Journal, 19, 719-722.

Bargh, J.A., 1994. The four horsemen of automaticity: awareness, intention, efficiency, and control in social cognition. In: Wyer Jr.R.S., Srull, T.K. (Eds.), Handbook of Social Cognition, 2nd ed. Erlbaum, Hillsdale, NJ, pp. 1-40.

Baumgartner, T., Heinrichs, M., Vonlanthen, A., Fischbacher, U., \& Fehr, E. (2008). Oxytocin shapes the neural circuitry of trust and trust adaptation in humans. Neuron, 58(4), 639-650.

Carretié, L., Mercado, F., Tapia, M., \& Hinojosa, J. A. (2001). Emotion, attention and the "negativity bias", studied through event-related potentials. International Journal of Psychophysiology, 41(1), 75-85.

Cardoso, C., Linnen, A. M., Joober, R., \& Ellenbogen, M. A. (2012). Coping style moderates the effect of intranasal oxytocin on the mood response to interpersonal stress. Experimental and Clinical Psychopharmacology, 20(2), 84.

Colonnello, V., Chen, F. S., Panksepp, J., \& Heinrichs, M. (2013). Oxytocin sharpens self-other perceptual boundary. Psychoneuroendocrinology, 38 (12), 2996-3002.

Decety, J., \& Jackson, P. L. (2004). The functional architecture of human empathy. Behavioral and Cognitive Neuroscience Reviews, 3(2), 71-100.

Decety, J., \& Sommerville, J. A. (2003). Shared representations between self and other: a social cognitive 
432

433

434

435

436

437

438

439

440

441

442

443

444

445

446

447

448

449

450

451

452

453

454

455

456

457

458

459

460

461

462

463

464

465

466

467

neuroscience view. Trends in Cognitive Sciences, 7(12), 527-533.

De Vignemont, F., \& Singer, T. (2006). The empathic brain: how, when and why? Trends in Cognitive Sciences, 10(10), 435-441.

Fischer-Shofty, M., Levkovitz, Y., \& Shamay-Tsoory, S. G. (2013). Oxytocin facilitates accurate perception of competition in men and kinship in women. Social Cognitive and Affective Neuroscience, 8, 313-317,

Fields, E. C., \& Kuperberg, G. R. (2012). It's all about you: an erp study of emotion and self-relevance in discourse. Neuroimage, 62(1), 562-574.

Huang, Y. X., \& Luo, Y. J. (2006). Temporal course of emotional negativity bias: an ERP study. Neuroscience Letters, 398(1-2), 91-96.

Jackson, P. L., Brunet, E., Meltzoff, A. N., \& Decety, J. (2006). Empathy examined through the neural mechanisms involved in imagining how I feel versus how you feel pain. Neuropsychologia, 44(5), 752761.

Jin, Y., Yan, K., Zhang, Y., Jiang, Y., Tao, R., \& Zheng, X. (2013). Gender differences in detecting unanticipated stimuli: an ERP study. Neuroscience Letters, 538, 38-42.

Kumsta, R., \& Heinrichs, M. (2012). Oxytocin, stress and social behavior: Neurogenetics of the human oxytocin system. Current Opinion in Neurobiology, 23, 1-6.

Li, Y., Li, F., Chen, J., \& Li, H. (2012). An erp study on the understanding of the distinction between real and apparent emotions. Neuroscience Letters, 529(1), 33-38.

Li, W., \& Han, S. (2010). Perspective taking modulates event-related potentials to perceived pain. Neuroscience Letters, 469(3), 328-332.

Liu, Y., Sheng, F., Woodcock, K. A., \& Han, S. (2013). Oxytocin effects on neural correlates of self-referential processing. Biological Psychology, 94(2), 380-387.

Luo, P., Wang, J., Jin, Y., Huang, S., Xie, M., Deng, L., ... \& Jiang, Y. (2015). Gender differences in affective sharing and self-other distinction during empathic neural responses to others' sadness. Brain Imaging and Behavior, 9(2), 312-322.

Ma, Y., Shamay-Tsoory, S., Han, S., \& Zink, C. F. (2016). Oxytocin and social adaptation: insights from neuroimaging studies of healthy and clinical populations. Trends in Cognitive Sciences, 20(2), 133-145.

O’Brien, E., Konrath, S. H., Grühn, D., \& Hagen, A. L. (2013). Empathic concern and perspective taking: Linear and quadratic effects of age across the adult life span. Journals of Gerontology Series B: Psychological Sciences and Social Sciences, 68(2), 168-175.

Pfundmair, M., Rimpel, A., Duffy, K., \& Zwarg, C. (2018). Oxytocin blurs the self-other distinction implicitly but not explicitly. Hormones and Behavior, 98, 115-120

Preckel, K., Scheele, D., Kendrick, K. M., Maier, W., \& Hurlemann, R. (2014). Oxytocin facilitates social approach behavior in women. Frontiers in Behavioral Neuroscience, 8, 191.

Quirin, M., Kuhl, J., \& Düsing, R. (2011). Oxytocin buffers cortisol responses to stress in individuals with impaired emotion regulation abilities. Psychoneuroendocrinology, 36(6), 898-904.

Peer] reviewing PDF | (2020:04:48012:2:0:NEW 25 Sep 2020) 
468

469

470

471

472

473

474

475

476

477

478

479

480

481

482

483

484

485

486

487

488

489

490

491

492

493

494

495

496

497

498

499

500

501

502

503

Sauer, C., Montag, C., Reuter, M., \& Kirsch, P. (2013). Imaging oxytocin $\times$ dopamine interactions: an epistasis effect of CD38 and COMT gene variants influences the impact of oxytocin on amygdala activation to social stimuli. Frontiers in Neuroscience, 7, 45.

Schulte-Rüther, M., Markowitsch, H. J., Shah, N. J., Fink, G. R., \& Piefke, M. (2008). Gender differences in brain networks supporting empathy. NeuroImage, 42(1), 393-403.

Schupp, H. T., Cuthbert, B. N., Bradley, M. M., Cacioppo, J. T., Ito, T., \& Lang, P. J. (2000). Affective picture processing: the late positive potential is modulated by motivational relevance. Psychophysiology, 37(2), 257-261.

Seidel, E. M., Habel, U., Kirschner, M., Gur, R. C., \& Derntl, B. (2010). The impact of facial emotional expressions on behavioral tendencies in women and men. Journal of Experimental Psychology: Human Perception and Performance, 36(2), 500.

Sheng, F, \& Han, S. (2012). Manipulations of cognitive strategies and intergroup relationships reduce the racial bias in empathic neural responses. Neuroimage, 61(4), 786-797.

Sheng, F., Liu, Q., Li, H., Fang, F., \& Han, S. (2014). Task modulations of racial bias in neural responses to others' suffering. NeuroImage, 88, 263-270.

Striepens, N., Kendrick, K. M., Hanking, V., Landgraf, R., Wüllner, U., Maier, W., \& Hurlemann, R. (2013). Elevated cerebrospinal fluid and blood concentrations of oxytocin following its intranasal administration in humans. Scientific Reports, 3, 3440.

Suh, D. H., Hong, J. S., Lee, D. H., Gonnella, J. S., \& Hojat, M. (2012). The Jefferson Scale of Physician Empathy: a preliminary psychometric study and group comparisons in Korean physicians. Medical Teacher, 34(6), e464-e468.

Theodoridou, A., Penton-Voak, I. S., \& Rowe, A. C. (2013). A direct examination of the effect of intranasal administration of oxytocin on approach-avoidance motor responses to emotional stimuli. PLoS One, 8 , $\mathrm{e} 58113$

Van Strien, J. W., De Sonneville, L. M. J., \& Franken, I. H. A. (2010). The late positive potential and explicit versus implicit processing of facial valence. Neuroreport, 21(9), 656-661.

Yuan, J., Zhang, Q., Chen, A., Li, H., Wang, Q., Zhuang, Z., \& Jia, S. (2007). Are we sensitive to valence differences in emotionally negativestimuli? Electrophysiological evidence from an ERP study. Neuropsychologia, 45(12), 2764-2771.

Yue, T., Jiang, Y., Yue, C., \& Huang, X. (2017). Differential Effects of Oxytocin on Visual Perspective Taking for Men and Women. Frontiers in Behavioral Neuroscience, 11, 228.

Zhang, F. F., Dong, Y., Wang, K., Zhan, Z., \& Xie, L. (2010). Reliability and validity of the Chinese version of the Inter-personal reactivity index-C. Chinese Journal of Clinical Psychology. 18, 155-157.

Zhao, W., Yao, S., Li, Q., Geng, Y., Ma, X., Luo, L., ... \& Kendrick, K. M. (2016). Oxytocin blurs the self-other distinction during trait judgments and reduces medial prefrontal cortex responses. Human Brain Mapping, 37(7), 2512-2527.

Peer] reviewing PDF | (2020:04:48012:2:0:NEW 25 Sep 2020) 
504

505

506

507

508

509

510

511

512

513

514

515

516

517

518

519

520

521

522

523

524

525

526

527

528
Table 1 Overall results of the ANOVAs of P2 (150-200 ms) and LPC (400-600 ms) with treatment type as the between-participants factor and task, facial expression, brain regions, and laterality as the withinparticipants factors.

${ }^{* * * *} p<0.01,{ }^{* *} p<0.01,{ }^{*} p<0.05$, , non-significant

Figure 1: CONSORT flow diagram of the participants

Figure 2 (A-L): Grand-average ERP waveforms from $\mathrm{Fz}, \mathrm{Cz}$, and Pz channels, elicited by emotional faces in the OXT and PLC groups for the other-task and the self-task

Figure 3 (A) Comparison of means and standard errors of P2 amplitudes for ERP statistics at 150-200 ms between the OXT and PLC groups for the other-task and the self-task. (B-E) Topographic maps elicited by the other- and the self-task in the OXT and PLC groups during the 150-200 ms time window. (F) The difference waves of P2 between the OXT and PLC groups for the other- and the self-task $\left({ }^{*} p<\right.$ 0.05).

Figure 4 (A) Comparison of means and standard errors of LPC amplitude for ERP statistics at 400-600 ms between the OXT and PLC groups for the other-task and the self-task. (B-E) Topographic maps elicited by the other-task and the self-task in the OXT and PLC groups during the 400-600 ms time window. (F) The difference waves of the LPC between the OXT and PLC groups for the other- and self$\operatorname{task}\left({ }^{* *} p<0.01,{ }^{* * *} p<0.001\right)$. 


\section{Table $\mathbf{1}$ (on next page)}

Overall results of ANOVA on P2 (150-200 ms) and LPC (400-600 ms) . 


\begin{tabular}{|c|c|c|c|c|}
\hline \multirow{2}{*}{ Source of variations } & \multicolumn{2}{|c|}{$\mathrm{P} 2$} & \multicolumn{2}{|c|}{ LPC } \\
\hline & $F$ & $\eta_{p}^{2}$ & $F$ & $\eta_{p}^{2}$ \\
\hline treatment type & - & - & - & - \\
\hline task & - & - & - & - \\
\hline facial expression & 4.11 & $0.10^{*}$ & 48.24 & $0.56^{* * *}$ \\
\hline brain regions & - & - & 73.40 & $0.66^{* * *}$ \\
\hline laterality & 9.31 & $0.20^{* * *}$ & 7.59 & $0.17^{* *}$ \\
\hline treatment type $\times$ task & - & - & 10.16 & $0.21^{* *}$ \\
\hline treatment type $\times$ facial expression & - & - & - & - \\
\hline treatment type $\times$ brain regions & - & - & - & - \\
\hline treatment type $\times$ laterality & - & - & - & - \\
\hline task $\times$ facial expression & - & - & - & - \\
\hline task $\times$ brain regions & 6.77 & $0.13^{*}$ & - & - \\
\hline task $\times$ laterality & - & - & - & - \\
\hline facial expression $\times$ brain regions & - & - & - & - \\
\hline facial expression $\times$ laterality & - & - & - & - \\
\hline brain regions $\times$ laterality & - & - & 10.37 & $0.21^{* * *}$ \\
\hline treatment type $\times$ task $\times$ facial expression & 5.99 & $0.14^{*}$ & 6.49 & $0.15^{*}$ \\
\hline treatment type $\times$ task $\times$ brain regions & - & - & - & - \\
\hline treatment type $\times$ task $\times$ laterality & 5.24 & $0.12^{* *}$ & - & - \\
\hline task $\times$ facial expression $\times$ brain regions & - & - & 7.36 & $0.16^{* * *}$ \\
\hline task $\times$ brain regions $\times$ laterality & 10.33 & $0.22^{* * *}$ & - & - \\
\hline facial expression $\times$ brain regions $\times$ laterality & 9.56 & $0.21^{* * *}$ & - & - \\
\hline treatment type $\times$ task $\times$ facial expression $\times$ brain regions & - & - & 2.83 & $0.07^{*}$ \\
\hline treatment type $\times$ task $\times$ facial expression $\times$ laterality & - & - & - & - \\
\hline task $\times$ facial expression $\times$ brain regions $\times$ laterality & 4.70 & $0.11^{*}$ & - & - \\
\hline treatment type $\times$ facial expression $\times$ brain regions $\times$ laterality & 5.90 & $0.14^{* *}$ & - & - \\
\hline
\end{tabular}


laterality

1 
Figure 1

\section{CONSORT flow diagram of the participants}

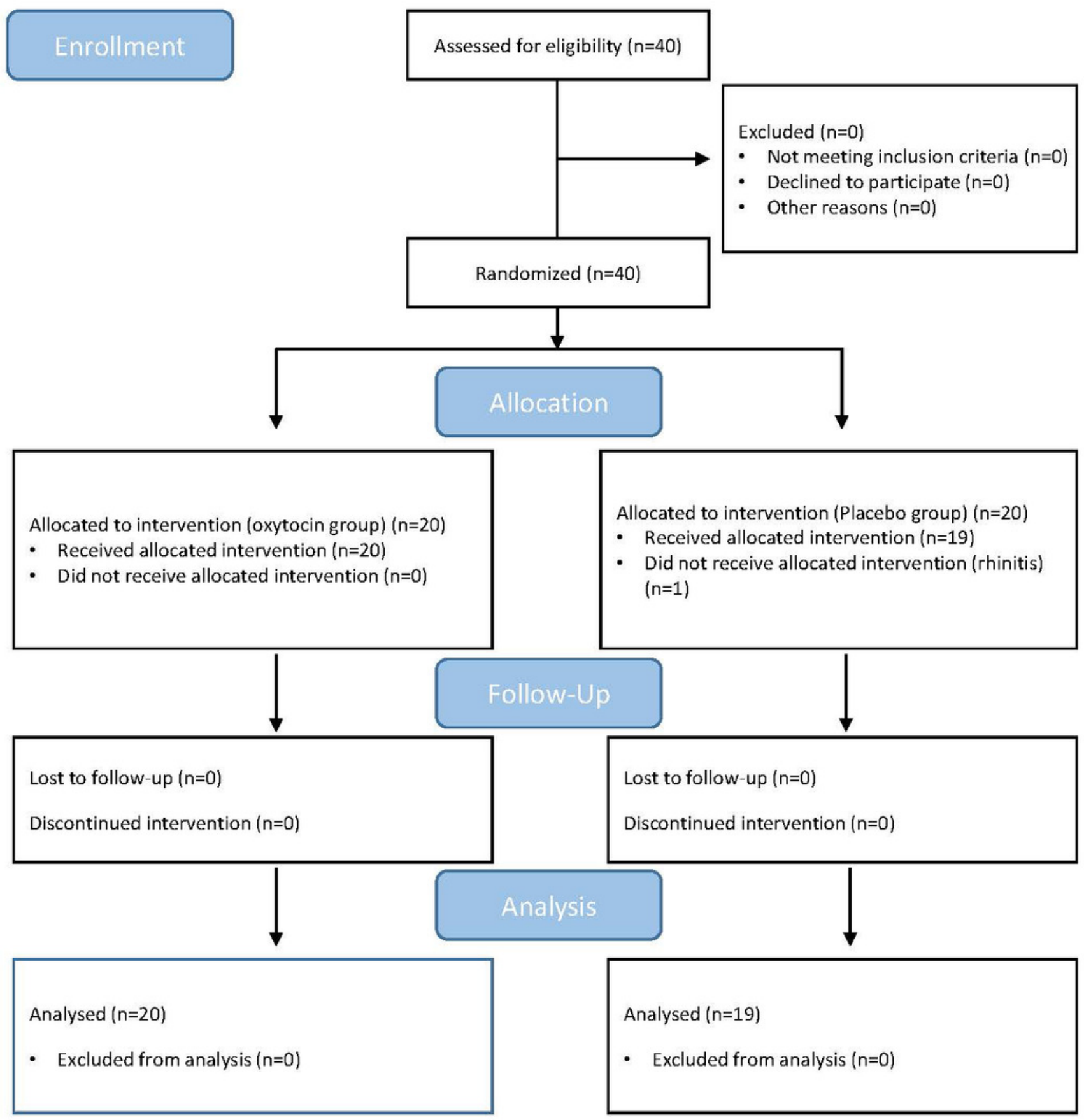


Figure 2

(A-L): Grand-average ERP waveforms from $\mathrm{Fz}, \mathrm{Cz}$ and Pz channels, elicited by emotional faces in the OXT and PLC groups for the other-task and the self-task 


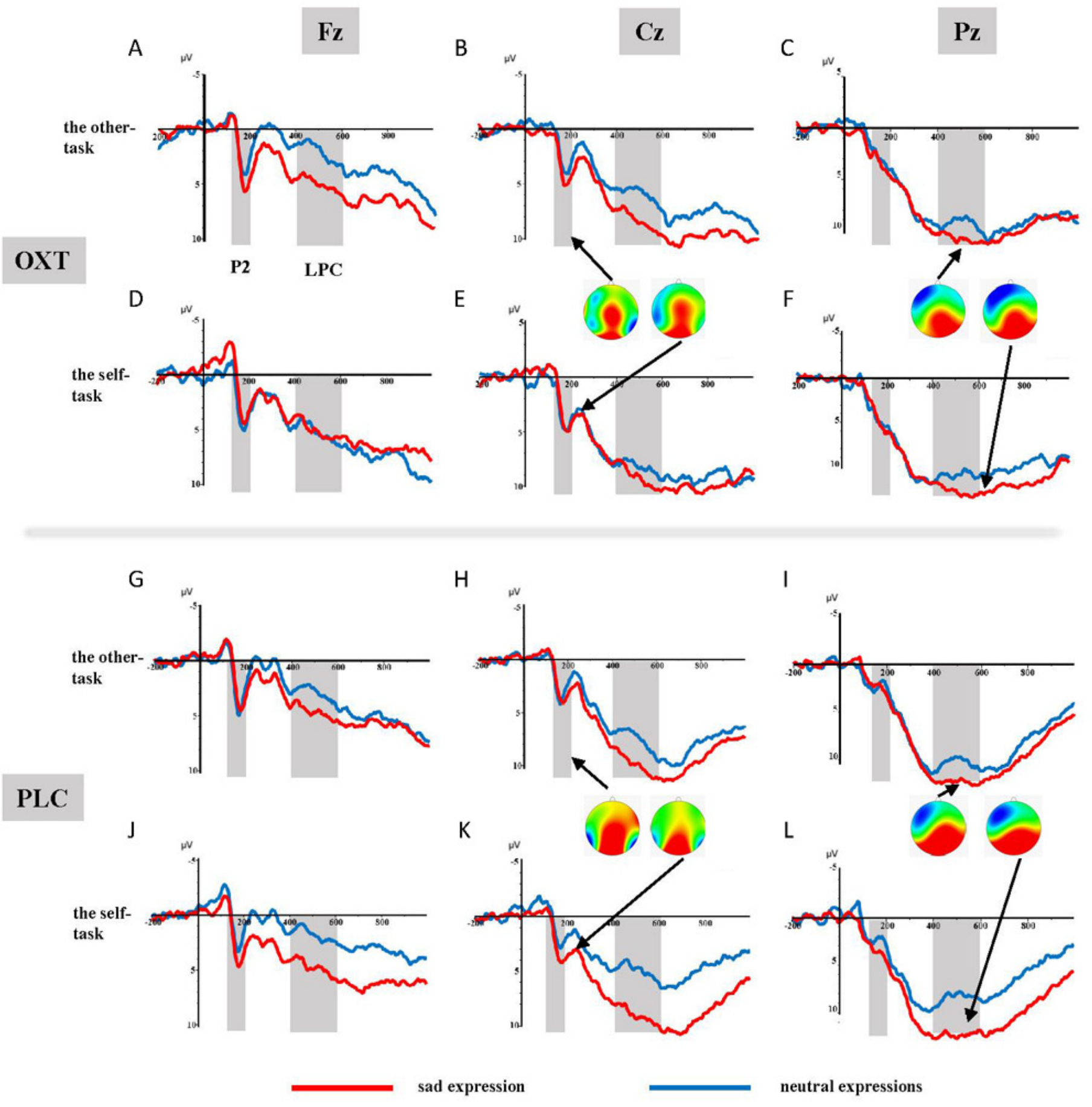


Figure 3

(A) Comparison of means and standard errors of P2 amplitudes. (B-E) Topographic maps elicited by the other- and the self-task. (F) The difference waves of P2 between the OXT and PLC groups. 


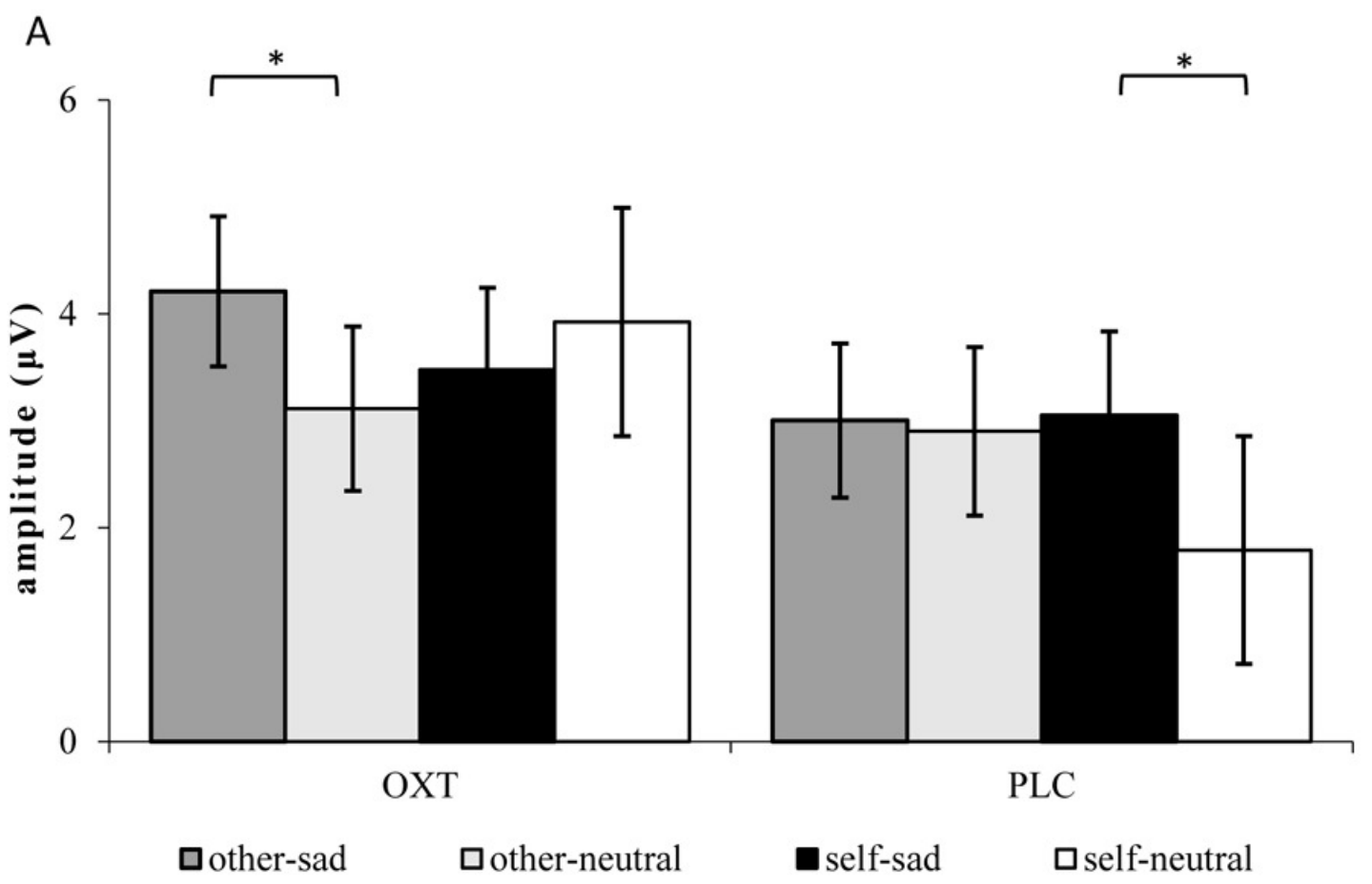

B

C

D

E
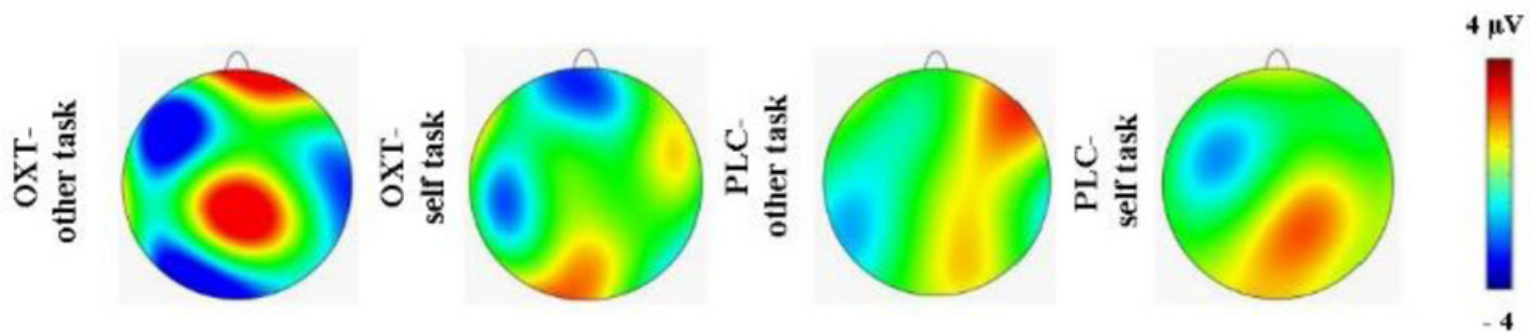

$\mathrm{F}$

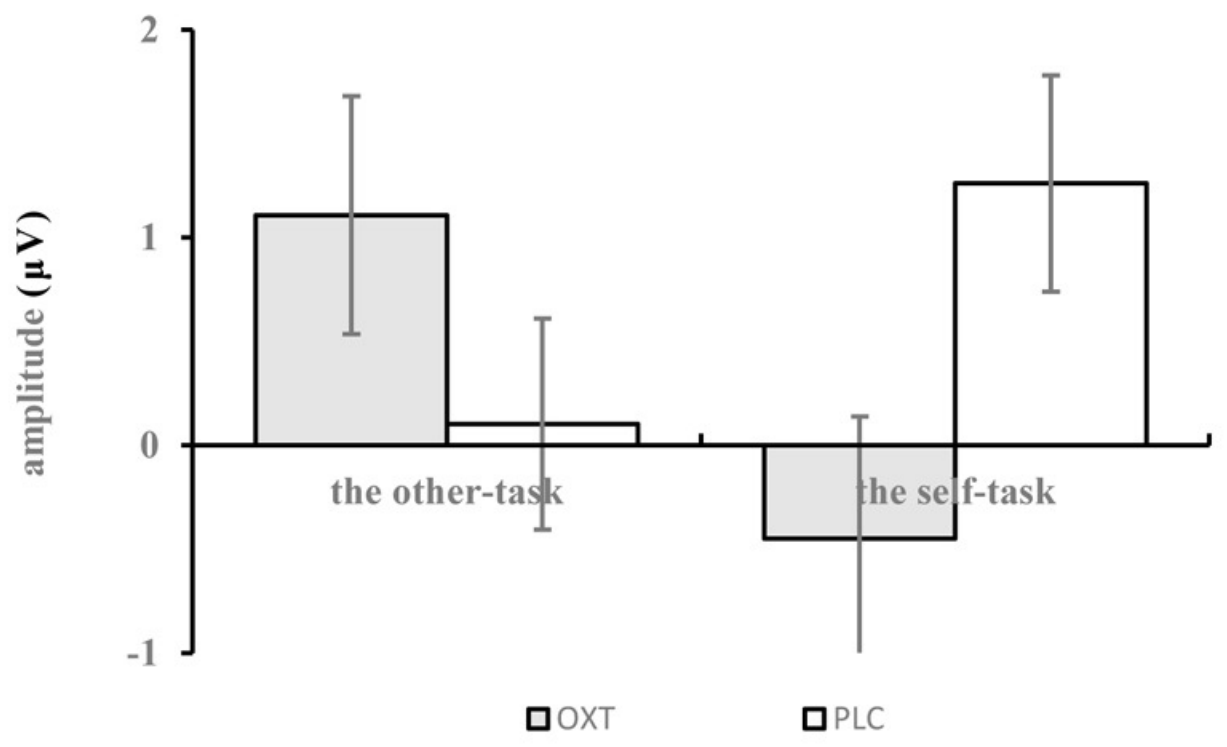


Figure 4

(A) Comparison of means and standard errors of LPC. (B-E) Topographic maps in the OXT and PLC groups. (F) The difference waves of the LPC between the OXT and PLC groups. 

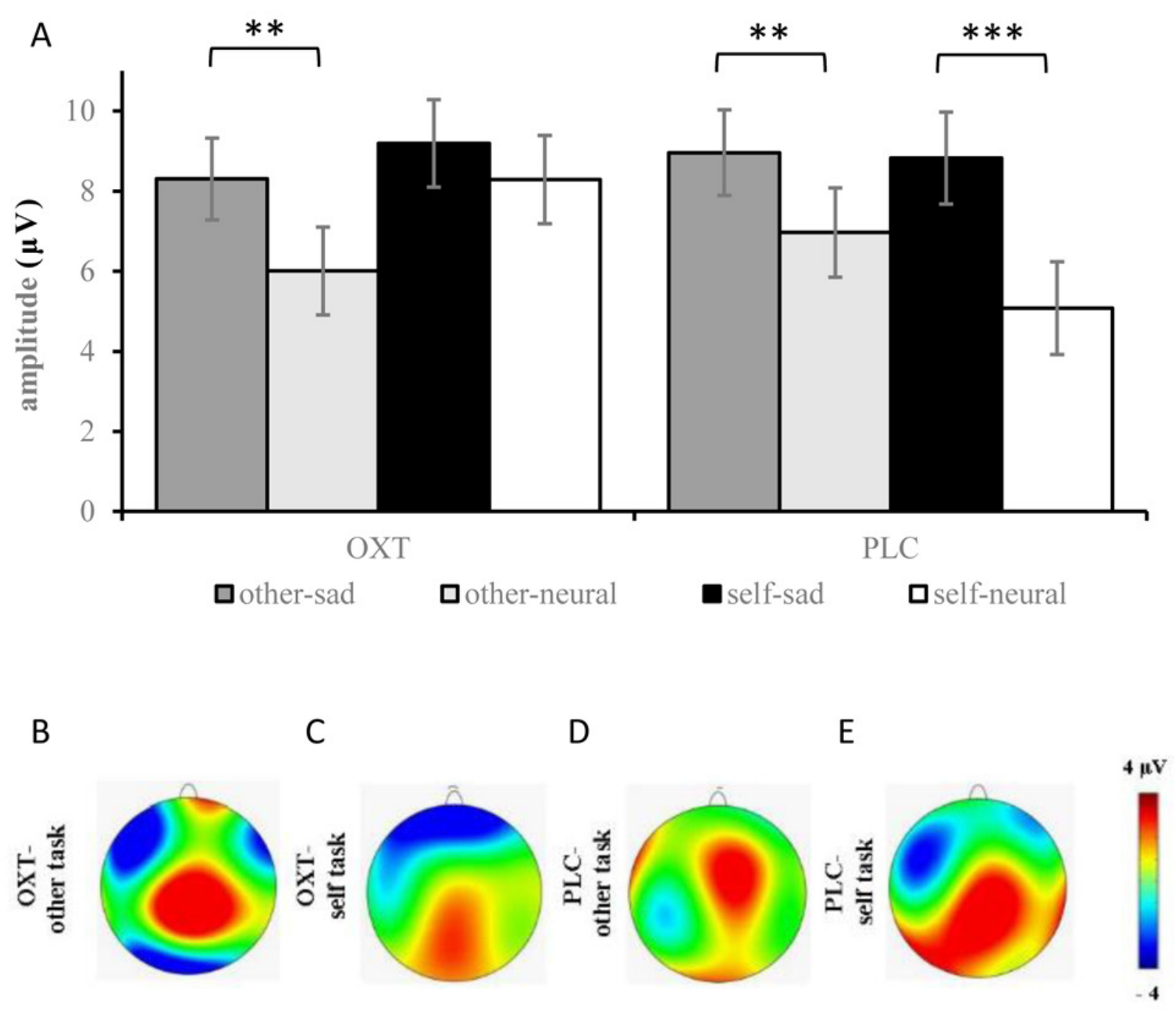

F

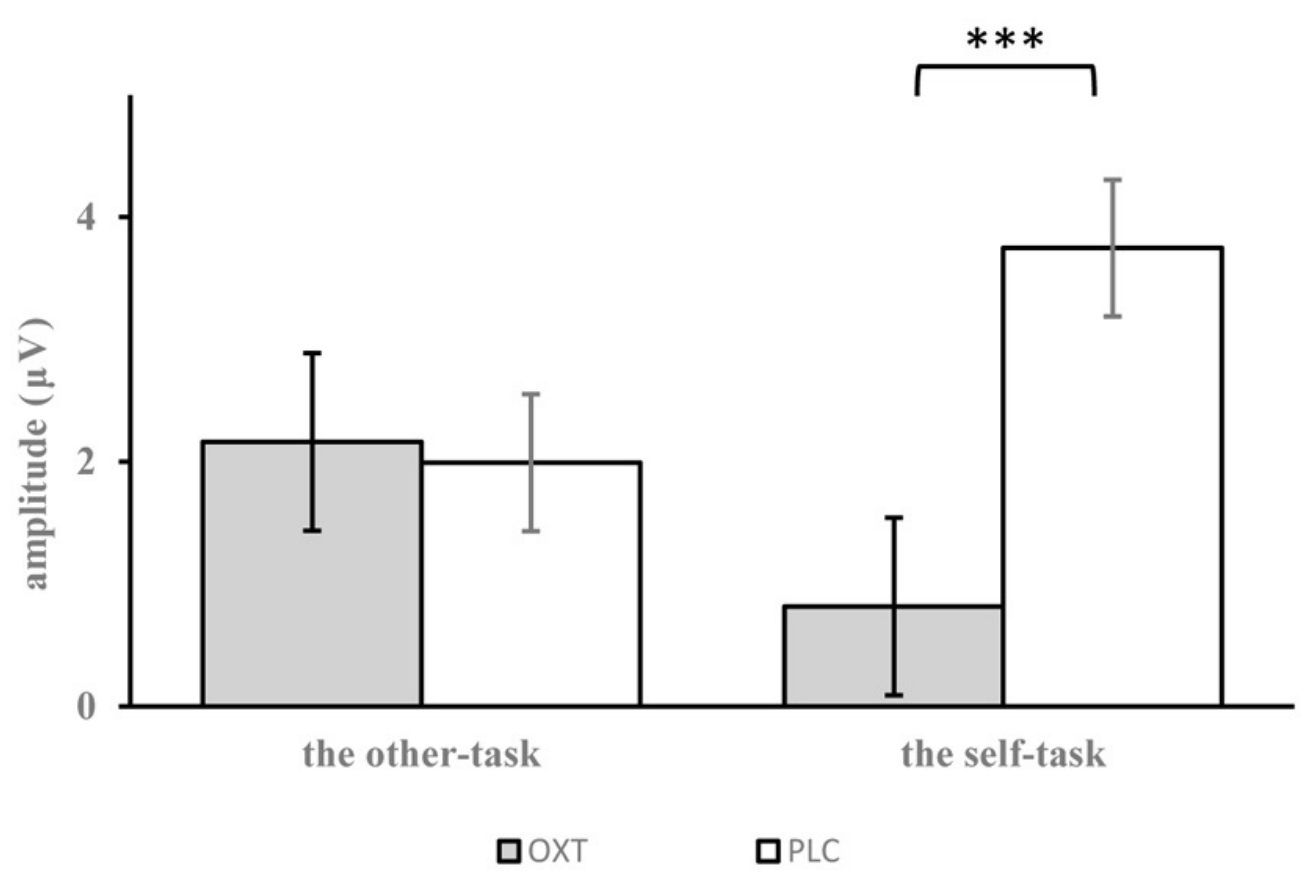

